\title{
On the Kobayashi-Royden metric for ellipsoids
}

\author{
L. D. Kay ${ }^{\star}$ \\ Department of Mathematics, University of Michigan, Ann Arbor, MI 48109, USA
}

Received January 29, 1990

The Kobayashi indicatrix (infinitesimal unit ball) of a domain in $\mathbb{C}^{n}$ is known to be a biholomorphic invariant. In particular, if a domain is biholomorphic to a ball, then the indicatrix is the ball. Until the recent deep results of Lempert [4], it was not known to what extent the indicatrix characterizes the domain. Sibony had shown earlier that the indicatrix of any pseudoconvex circular domain is the domain itself [10]; hence the indicatrix determines such domains up to biholomorphism. This is not true in general. Lempert has shown that, for smoothly bounded strictly convex domains, three invariants determine the domain up to biholomorphism and a linear change of variables at a base point. These invariants consist of not only the Kobayashi indicatrix but also a quadratic form and a hermitian form on a certain vector bundle. In two dimensions, there is an open set in a Frechet space, corresponding to domains not biholomorphic to the ball but with indicatrix the ball.

The result of this paper is that, for ellipsoids, if the Kobayashi indicatrix is the ball then the ellipsoid is biholomorphic to the ball. This is proved in the following equivalent form: if an ellipsoid symmetric about 0 is not biholomorphic to the ball, then its infinitesimal Kobayashi metric at 0 is not hermitian.

In this language, our result answers a question posed by Reiffen in [7] about the differential-geometric nature of the Carathéodory metric for ellipsoids. By two results of Lempert ([3] and [2]), for strictly convex domains the Caratheódory and Kobayashi metrics coincide and determine a Finsler metric. One now sees that, for ellipsoids, the metric is not hermitian and hence, by a theorem of Reiffen [7], not Riemannian.

We prove that the infinitesimal Kobayashi metric at 0 is not hermitian by proving that the parallelogram law fails to hold. An ellipsoid $\mathscr{E}$ may be written (via a linear change of coordinates) as $\sum\left|z_{j}\right|^{2}+\lambda_{j} \operatorname{Re}\left(z_{j}^{2}\right)<, 0 \leqq \lambda_{j}<1$. By a

To the memory of Melissa Knapp

* Current address: Department of Mathematics, Virginia Polytechnic Institute and State University, Blacksburg, VA 24061-0123, USA 
result of Webster [12], if $\mathscr{E}$ is not biholomorphic to the ball then some parameter $\lambda_{j 1}=\lambda$ is nonzero. Fixing $j_{2} \neq j_{1}$ with corresponding $\lambda_{j_{2}}=\mu$, we show that the parallelogram law fails for the vectors $i t \partial / \partial z_{j 1}$ and $\partial / \partial z_{j 2}$ if $\mu=0$, and $\partial / \partial z_{j_{1}}$ and $i t \partial / \partial z_{j_{2}}$ if $\mu \neq 0 . t$ is either $\frac{1}{2}, 1$, or 2 , depending on the ratio $\mu / \lambda$.

In the $z_{j_{1}}$-and $z_{j_{2}}$-axis directions, symmetriy and Lempert's uniqueness result [2] are used to reduce the problem of determining Kobayashi extremal disks from $n$ dimensions to one. In $\mathbb{C}^{1}$ the Kobayashi extremal disks must coincide with the inverse of the Riemann mapping function for ellipses. Off the axes, the extremal disks are not calculated exactly. We consider competitors lying in complex lines, thus reducing the problem again to one dimension, and use the Riemann maps. The Kobayashi norm (or a competitor for the norm) of a vector in the tangent space at zero is the derivative at zero of the inverse of the Riemann mapping function. This derivative involves an elliptic integral and the modulus of a Jacobi elliptic function, and the classical elliptic function technology is used to express norms (or competitors) as series in terms of certain mapping parameters $\rho$ of the ellipses. These $\rho$ are algebraic functions of $\lambda$ and $\mu$.

Hence showing that the parallelogram law does not hold is equivalent to showing that one series is greater than another. In Sect. 1 the problem is reduced to this stage. In Sect. 2 we compare rates of growth of the series and in Sect. 3 their initial terms. The lemmas of these sections provide the induction step and the hypothesis for an easy induction argument in Sect. 4, which shows that one series dominates the other term-by-term and completes the proof of the theorem. Finally, we sketch why the theorem as stated is equivalent to the assertion about indicatrices.

The author wishes to thank her thesis advisor, Dan Burns, who suggested using the parallelogram law to study the Kobayashi metric and suggested considering ellipsoids. The author also wishes to thank Bill Floyd, who wrote a computer program which made possible the study of numerous examples.

\section{Reduction to a comparison of series}

We first introduce some definitions and notation. $\Delta$ denotes the unit disk $\{\zeta \in \mathbb{C}:|\zeta|<1\}$. For a strictly convex domain $D$ in $\mathbb{C}^{n}$, a holomorphic function $f: \Delta \rightarrow D$ will be called extremal with respect to $z \in D$ and $v \in T_{z}(D)$ if $f(0)=z, f^{\prime}(0)=\alpha v$, with $\alpha>0$, and for any holomorphic $g: \Delta \rightarrow D$ such that $g(0)=z, g^{\prime}(0)=\tilde{\alpha} v(\tilde{\alpha}>0)$, we have $\tilde{\alpha} \leqq \alpha$. In this context the Kobayashi-Royden infinitesimal metric at $z$ is $F_{D}(z, v)=1 / \alpha$. Existence and uniqueness of $f$ for such $D$ are due to Lempert [2]; in general $F_{D}$ is given as an infimum, [8]. That $F_{D}$ is in fact a norm on the tangent spaces of strictly convex $D$ also follows from Lempert [2]. Throughout this paper the base point $z=0$, the domain is a fixed ellipsoid $\mathscr{E}$, and we write $\|v\|$ for $F_{\mathscr{E}}(0, v) . f(\Delta)$ will be called an extremal disk. The Kobayashi indicatrix at 0 is $I_{0}=\left\{v: F_{D}(0, v)<1\right\}$; it will not be referred to until Sect. 4.

An ellipsoid is the bounded domain in $\mathbb{C}^{n}$ with boundary the real $(2 n-1)$ dimensional hypersurface described by

$$
\sum_{j=1}^{n} a_{j} x_{j}^{2}+b_{j} y_{j}^{2}=1, \quad a_{j} \geqq b_{j}>0, \quad z_{j}=x_{j}+i y_{j} .
$$


Following Webster [12] we make the linear change of coordinates $z_{j} \Rightarrow \sqrt{\frac{2}{a_{j}+b_{j}}} \cdot z_{j}$ to rewrite the above in the form

$$
\sum_{j=1}^{n}\left|z_{j}\right|^{2}+\lambda_{j} \operatorname{Re}\left(z_{j}\right)^{2}=1, \quad \lambda_{j}=\frac{a_{j}-b_{j}}{a_{j}+b_{j}}, \quad 0 \leqq \lambda_{j}<1 .
$$

Let $\mathscr{E}$ be an ellipsoid not biholomorphic to the ball in $\mathbb{C}^{n}$, with boundary defined by $\left({ }^{*}\right)$. Then by Webster's ellipsoid classification result [12] some $\lambda_{j}$, say $\lambda_{j_{1}}$, is nonzero. Fix $j_{2} \neq j_{1}$. If $\lambda_{j_{2}} \neq 0$, w show that the parallelogram law

$$
2\left(\|u\|^{2}+\|v\|^{2}\right)=\|u+v\|^{2}+\|u-v\|^{2}
$$

fails for the vectors $u=\frac{\partial}{d z_{j_{1}}}$ and $v=i t \frac{\partial}{\partial z_{j_{2}}}$; $t$ is a real number which will vary with the ratio $\lambda_{j 2} / \lambda_{j 1}$. If $\lambda_{j 2}=0$, we use the vectors $\frac{1}{2} i \frac{\partial}{\partial z_{j 1}}$ and $\frac{\partial}{\partial z_{j 2}}$.

Under automorphisms of $\mathscr{E}$, Kobayashi extremal disks are mapped to Kobayashi extremal disks. It follows from Lempert's uniqueness theorem [2, théorème 2] that the extremal disk in the $z_{j_{1}}$-direction is invariant under the automorphism $z_{j 1} \mapsto z_{j 1}, z_{j} \mapsto-z_{j}$ for $j \neq j_{1}$. Hence the disk lies in the $z_{j 1}$-axis. Similarly, the extremal disk in the $z_{j 2}$-direction lies in the $z_{j 2}$-axis.

With $u$ and $v$ as above, the automorphism $z_{j_{2}} \mapsto-z_{j 2}, z_{j} \mapsto z_{j}$ if $j \neq j_{2}$ interchanges $u+v$ and $u-v$. Hence $\|u+v\|=\|u-v\|$. So we need only show that $\|u\|^{2}+\|v\|^{2}=\|u+v\|^{2}$ fails to hold.

If there were automorphisms of $\mathscr{E}$ fixing the directions $u+v$ and $u-v$, we could conclude, as in the axis directions, that the extremal disks each lay in a complex line. Since this is not true in general, we estimate the extremal disks.

Note at this point that, since the vectors in question depend on only two of the standard basis vectors $\left\{\frac{\partial}{\partial z_{1}}, \ldots, \frac{\partial}{\partial z_{n}}\right\}$ for $T_{0}^{1,0}(D)$, the argument is essentially two-dimensional. To simplify notation we suppress lists of zeros and work in $\mathbb{C}^{2}$, with $z$ for $z_{j 1}, w$ for $z_{j_{2}}, \lambda$ for $\lambda_{j_{1}}$, and $\mu$ for $\lambda_{j_{2}}$.

Let $g$ denote the extremal disk in the direction $u+v$. Then $g^{\prime}(0)$ $=\alpha(u+v), \alpha>0$ maximal. If $\tilde{g}$ is a holomorphic map from $\Delta$ to $\mathscr{E}$ with $\tilde{g}^{\prime}(0)=\tilde{\alpha}(u+v)$, then $\tilde{\alpha} \leqq \alpha$ and $\|u+v\|=\frac{1}{\alpha} \leqq \frac{1}{\tilde{\alpha}}$. In particular this holds for a competitor $\tilde{g}$ lying in a complex line. We will compute one such $\tilde{g}$. Writing $\|u+v\|_{-}=\frac{1}{\tilde{\alpha}}$, we will show that

$$
\|u\|^{2}+\|v\|^{2}>\|u+v\|^{2} \sim
$$

It follows from Schwarz's lemma that, for a bounded simply connected domain in $\mathbb{C}$, the Kobayashi extremal function is the inverse of the Riemann mapping function. Since the Riemann map for ellipses is known, we may compute $\left\|\frac{\partial}{\partial z}\right\|$ and $\left\|\frac{\partial}{\partial w}\right\|$. 
The interior of the ellipse $\frac{x^{2}}{a^{2}}+\frac{y^{2}}{b^{2}}=1, a^{2}-b^{2}=1, w=x+i y$, is mapped conformally onto the unit disk $\Delta$ by the function

$$
z=f(w)=\sqrt{k(\rho)} \operatorname{sn}\left(\frac{2 K(\rho)}{\pi} \sin ^{-1} w ; \rho\right), \quad \rho=\left(\frac{a-b}{a+b}\right)^{2} .
$$

[5, pp. 295-296]. Sn is a Jacobi elliptic function which may be defined by means of its inverse,

$$
\operatorname{sn}^{-1}(z)=\int_{0}^{z} \frac{d \zeta}{\left(1-\zeta^{2}\right)\left(1-k^{2} \zeta^{2}\right)}
$$

It appears here via the Schwarz-Christoffel formula mapping a rectangle to the upper halfplane. $\rho$ is, up to constant factors in the exponent, the exponentiated ratio of the sides of the rectangle. Therefore the inverse of the Riemann map for an ellipse with foci at $( \pm c, 0)$ is given by $F(z)=c f^{-1}(z)$. Since $f(0)=0, F^{\prime}(0)=c\left(f^{-1}\right)^{\prime}(0)$.

$$
f^{-1}(z)=\sin \left\{\frac{\pi}{2 K} \operatorname{sn}^{-1}\left(\frac{z}{\sqrt{k}} ; \rho\right)\right\},
$$

so $\left(f^{-1}\right)^{\prime}(0)=\frac{\pi}{2 K \sqrt{k}}$.

We consider first the case $\mu \neq 0$. To find $\left\|\frac{\partial}{\partial z}\right\|^{2}$, consider the ellipse $E_{1}$ determined by the intersection of $\mathscr{E}$ with the $z$-axis given by $|z|^{2}+\lambda \operatorname{Re}\left(z^{2}\right)=1$, or $(1+\lambda) x^{2}+(1-\lambda) y^{2}=1$. We rotate to get the foci on the $x$-axis and scale to get them at $( \pm 1,0) \cdot c^{2}=\frac{2 \lambda}{1-\lambda^{2}}$, so the scaling map is $c=\sqrt{\frac{2 \lambda}{1-\lambda^{2}}}$. The new ellipse is $\frac{2 \lambda}{1+\lambda} x^{2}+\frac{2 \lambda}{1-\lambda} y^{2}=1$. The corresponding $\rho$ is $\rho_{1}=\frac{\left(1-\sqrt{1-\lambda^{2}}\right)^{2}}{\lambda^{2}}$. The Kobayashi map is $\zeta \mapsto(F(\zeta), 0)$, where $F=\sqrt{\frac{2 \lambda}{1-\lambda^{2}}}$. (inverse of the Riemann map for $E_{1}$ ).

and

$$
F^{\prime}(0)=\sqrt{\frac{2 \lambda}{1-\lambda^{2}}} \cdot \frac{\pi}{2 K_{1} \sqrt{k_{1}}}
$$

$$
\left\|\frac{\partial}{\partial z}\right\|^{2}=\frac{\left|\frac{\partial}{\partial z}\right|^{2}}{\left|F^{\prime}(0)\right|^{2}}=\frac{1-\lambda^{2}}{2 \lambda} \cdot \frac{4}{\pi^{2}} K_{1}^{2} k_{1}, \quad K_{1}=K\left(\rho_{1}\right), \quad k_{1}=k\left(\rho_{1}\right) .
$$

Similarly, considering the ellipse determined by the intersection of $\mathscr{E}$ with the $w$-axis, we find that $\rho_{2}=\frac{\left(1-\sqrt{1-\mu^{2}}\right)^{2}}{\mu^{2}}$ and

$$
\begin{gathered}
\left\|i t \frac{\partial}{\partial w}\right\|^{2}=t^{2}\left\|\frac{\partial}{\partial w}\right\|^{2}=t^{2} \cdot \frac{1-\mu^{2}}{2 \mu} \cdot \frac{4}{\pi^{2}} K_{2}^{2} k_{2}, \\
K^{2}=K\left(\rho_{2}\right), \quad k_{2}=k\left(\rho_{2}\right) .
\end{gathered}
$$


In order to find $\left\|\frac{\partial}{\partial z}+i t \frac{\partial}{\partial w}\right\|_{\sim}$, consider the intersection of $\mathscr{E}$ with a complex line of the form $w=i t z, t>0$. This determines the ellipse

$$
E_{3}:|z|^{2}+|i t z|^{2}+\lambda \operatorname{Re}\left(z^{2}\right)+\mu \operatorname{Re}\left[(i t z)^{2}\right]=1
$$

or $\left[\left(1+t^{2}\right)-\left(\mu t^{2}-\lambda\right)\right] x^{2}+\left[\left(1+t^{2}\right)+\left(\mu t^{2}-\lambda\right)\right] y^{2}=1$. Note that the foci lie on the $x$-axis if $t^{2}>\frac{\lambda}{\mu}$; this will be satisfied for the values of $t$ to be selected. Let $r=\mu t^{2}-\lambda$ and $s=1+t^{2}$. Then the equation of the ellipse is $[s-r] x^{2}+[s+r] y^{2}=1$, $c^{2}=\frac{2 r}{s^{2}-r^{2}}$, the ellipse with foci at $( \pm 1,0)$ is $\frac{2 r}{s+r} x^{2}+\frac{2 r}{s-r} y^{2}=1$, and $p_{3}=\frac{s^{2}}{r^{2}}\left(1-\sqrt{1-\frac{r^{2}}{s^{2}}}\right)^{2}$. The map $\tilde{g}$ we use as competitor for the Kobayashi extremal is $\zeta \mapsto(G(\zeta), i t G(\zeta))$, where $G=\sqrt{\frac{2 r}{s^{2}-r^{2}}} \cdot$ (inverse of the Riemann map for $E_{3}$ ).

$$
\left\|\frac{\partial}{\partial z}+i t \frac{\partial}{\partial w}\right\|_{\sim}^{2}=\frac{\left|\frac{\partial}{\partial z}+i t \frac{\partial}{\partial w}\right|^{2}}{\left|\tilde{g}^{\prime}(0)\right|^{2}}=s \cdot \frac{1-\frac{r^{2}}{s^{2}}}{2 \frac{r}{s}} \frac{4}{\pi^{2}} K_{3}^{2} k_{3}
$$

Now, if $\mu=0$ we have $\mathscr{E}:|z|^{2}+|w|^{2}+\lambda \operatorname{Re}\left(z^{2}\right)<1$. As before $\left\|\frac{\partial}{\partial z}\right\|^{2}=\frac{1-\lambda^{2}}{2 \lambda} \cdot \frac{4}{\pi^{2}} K_{1}^{2} k_{1}$, so

$$
\left\|i t \frac{\partial}{\partial z}\right\|^{2}=t^{2} \frac{1-\lambda^{2}}{2 \lambda} \cdot \frac{4}{\pi^{2}} K_{1}^{2} k_{1} .
$$

The intersection of $\mathscr{E}$ and the $w$-axis is just $\Delta$, and

$$
\left\|\frac{\partial}{\partial w}\right\|^{2}=1 .
$$

To compute $\left\|i t \frac{\partial}{\partial z}+\frac{\partial}{\partial z}\right\|_{\sim}^{2}$, we consider the ellipse $E_{4}$ determined by the intersection of $\mathscr{E}$ with the line $z=i t w . \quad E_{4}$ is $|z|^{2}+|w|^{2}+\lambda \operatorname{Re}\left(z^{2}\right)=1$, or $\left(1+t^{2}-\lambda t^{2}\right) x^{2}+\left(1+t^{2}+\lambda t^{2}\right) y^{2}=1$. Let $p=\lambda t^{2}$ and $s=1+t^{2}$. The equation of the ellipse is $[s-p] x^{2}+[s+p] y^{2}=1, c^{2}=\frac{2 p}{s^{2}-p^{2}}$, the ellipse with foci at $( \pm 1,0)$ is $\frac{2 p}{s+p} x^{2}+\frac{2 p}{s-p} y^{2}=1$, and $\rho_{4}=\frac{s^{2}}{p^{2}}\left(1-\sqrt{1-\frac{p^{2}}{s^{2}}}\right)^{2}$. The competitor for the Kobayashi disk is the map $\tilde{h}: \zeta \mapsto(i t H(\zeta), H(\zeta))$, where $H=\sqrt{\frac{2 p}{s^{2}-p^{2}}} \cdot$ (inverse of the Riemann map for $E_{4}$ ).

$$
\left\|i t \frac{\partial}{\partial t}+\frac{\partial}{\partial w}\right\|_{\sim}^{2}=\frac{\left|i t \frac{\partial}{\partial z}+\frac{\partial}{\partial w}\right|^{2}}{\left|h^{\prime}(0)\right|^{2}}=s \cdot \frac{1-\frac{p^{2}}{s^{2}}}{2 \frac{p}{s}} \cdot \frac{4}{\pi^{2}} K_{4}^{2} k_{4} .
$$


All the norms computed above involve the quantity $\frac{4}{\pi^{2}} K^{2} k$, for which Jacobi $[1$, p. 160 , eq. (13)] found the following series expansion:

$$
\frac{4}{\pi^{2}} K^{2} k=4 \sum_{n=1}^{\infty} \rho^{n-\frac{1}{2}} \frac{1+\rho^{2 n-1}}{\left(1-\rho^{2 n-1}\right)^{2}} .
$$

(Cf. also $[11$, p. 108 , eq. CX (10)].)

\section{Comparison of growth rates}

Let

$$
G(\rho)=4 \sum_{n=1}^{\infty} \rho^{n-\frac{1}{2}} \frac{1+\rho^{2 n-1}}{\left(1-\rho^{2 n-1}\right)^{2}}
$$

and let $G_{n}$ denote the $n^{\text {th }}$ term of the series. Let $H_{n}(\rho)=G_{n+1}(\rho) / G_{n}(\rho)$.

Lemma 2.1. $H_{n}^{\prime}(\rho)>0$ for $\rho \in\left(0, \frac{1}{2}\right)$ and $n \geqq 2$.

Proof.

$$
\begin{gathered}
H_{n}(\rho)=\rho \cdot \frac{\left(1+\rho^{2 n+1}\right)}{\left(1-\rho^{2 n+1}\right)^{2}} \cdot \frac{\left(1-\rho^{2 n-1}\right)^{2}}{\left(1+\rho^{2 n-1}\right)} \text { and } \\
H_{n}^{\prime}(\rho)=\frac{1-\rho^{2 n-1}}{\left(1-\rho^{2 n+1}\right)^{3}\left(1+\rho^{2 n-1}\right)^{2}} P_{n}(\rho), \quad \text { where } \\
P_{n}(\rho)=-\rho^{8 n}+(6 n-3) \rho^{6 n+1}+(-6 n-3) \rho^{6 n-1}+2 n \rho^{4 n+2}-2 n \rho^{4 n-2} \\
+(6 n+3) \rho^{2 n+1}+(-6 n+3) \rho^{2 n-1}+1 .
\end{gathered}
$$

In order to show that $P_{n}(\rho)>0$, consider separately the cases $n=2$ and $n \geqq 3$. $P_{2}(0)>0, P_{2}\left(\frac{1}{2}\right)>0$, and $P_{2}^{\prime \prime}(\rho)<0$ for $\rho \in\left(0, \frac{1}{2}\right)$; hence $P_{2}(\rho)>0$. If $n \geqq 3$, group the terms of $P_{n}(\rho)$ pairwise (first and second, third and fourth, etc.). The positivity of each pair is easily checked for $\rho \in\left(0, \frac{1}{2}\right)$.

We now fix values for $t$. If $\mu=0$, we let $t=\frac{1}{2}$. If $\mu \neq 0$ we may assume by symmetry that $\mu \geqq \lambda$. Let $\mu=c \lambda$ and split the region $0<\lambda \leqq \mu<1$ in three: region I, where $1 \leqq c<1.6$; region II, where $1.6 \leqq c<10$; and region III, where $c \geqq 10$. In region I, let $t=2$; in region II, let $t=1$; and in region III, let $t=\frac{1}{2}$.

Define

$$
\rho(x)= \begin{cases}\frac{\left(1-\sqrt{1-x^{2}}\right)^{2}}{x^{2}}, & x \in(0,1) \\ 0, & x=0 .\end{cases}
$$

Then $\rho$ is a continuous function from $[0,1)$ to $[0,1)$ and, in the notation of Sect. 1 , $\rho_{1}=\rho(\lambda), \rho_{2}=\rho(\mu), \rho_{3}=\rho\left(\frac{r}{s}\right)$, and $\rho_{4}=\rho\left(\frac{p}{s}\right)$. Recall that $\lambda \in(0,1), \mu \in[0,1)$, 
$r=u t^{2}-\lambda, s=1+t^{2}$, and $p=t^{2} \lambda$. It is easily seen that $\frac{p}{s} \in[0,1)$ for all $t$ and that ${ }_{s}^{r} \in[0,1)$ for the values of $t$ selected above.

Lemma 2.2. The inequalities $\rho_{1}>\kappa \rho_{4}$ and $\rho_{2}>\kappa \rho_{3}$ are satisfied by the following values of $\kappa:$ for $\mu=0, \kappa=4$; for $\mu \neq 0, \kappa=4$ in regions $I I$ and $I I I$, and $\kappa=2.19$ in region $I$.

Proof. We abuse notation by writing " $\rho_{3}$ " in regions I, II, and III even though the explicit dependence on $\lambda$ and $c$ changes with $t$.

Consider first the case $\mu=0$. Then

$$
t=\frac{1}{2} \quad \rho_{4}=\frac{25}{\lambda^{2}}\left(1-\sqrt{1-\frac{\lambda^{2}}{25}}\right)^{2}, \quad \text { and } \quad \lim _{\lambda \downarrow 0} \rho_{1} / \rho_{4}=25 .
$$

For $\lambda \in(0,1), \rho_{1}=4 \rho_{4}$ if and only if

$$
\frac{1}{\lambda^{2}}\left(1-\sqrt{1-\lambda^{2}}\right)^{2}=\frac{100}{\lambda^{2}}\left(1-\sqrt{1-\frac{\lambda^{2}}{25}}\right)^{2},
$$

or, equivalently, $9 \lambda^{2}\left(\lambda^{2}+24\right)=0$. But the polynomial is positive for nonzero $\lambda$. It follows from $\lim _{\lambda \downarrow 0} \rho_{1} / \rho_{4}>4, \rho_{1} \neq 4 \rho_{4}$ for $\lambda \in(0,1)$, and continuity that $\rho_{1}>4 \rho_{4}$ for $\lambda \in(0,1)$.

The case $\mu \neq 0$ is similar, except that the $\rho$ 's now depend on both $\lambda$ and $c$. In each of regions I, II, and III, one shows that $\lim _{\lambda \downarrow 0} \rho_{2} / \rho_{3}$ is greater than the relevant $\kappa$ and that a polynomial in $\lambda$ and $c$ is positive. In regions II and III these polynomials are

$$
\begin{aligned}
P_{\mathrm{II}}(\lambda, c) & =\lambda^{2}\left[9 c^{2}(c-1)^{2} \lambda^{2}+48 c+16\right] \\
P_{\mathrm{III}}(\lambda, c) & =\lambda^{2}\left[9 c^{2}(c-4)^{2} \lambda^{2}+200 c^{2}+800 c+16(c-4)^{2}\right]
\end{aligned}
$$

Both are clearly positive for positive $c$ and nonzero $\lambda$, and the conclusion follows by continuity. The analysis in region $I$ is slightly more delicate and is deferred to the appendix.

Lemma 2.3. For $\rho \in(0,1 / \kappa), H_{n}(\kappa \rho)>H_{n}(\rho)$ for $n \geqq 2$ if $\kappa=4$ and for $n \geqq 3$ if $\kappa=2.19$.

Proof.

$$
\frac{H_{n}(\kappa \rho)}{H_{n}(\rho)}=\kappa\left(\frac{1-(\kappa \rho)^{2 n-1}}{1-(\kappa \rho)^{2 n+1}}\right)^{2} \cdot \frac{1+(\kappa \rho)^{2 n+1}}{1+(\kappa \rho)^{2 n-1}} \cdot \frac{1+\rho^{2 n-1}}{1+\rho^{2 n+1}} \cdot\left(\frac{1-\rho^{2 n+1}}{1-\rho^{2 n-1}}\right)^{2} .
$$

Since $\rho<1 / \kappa<1$, the last two factors are each greater than 1 . We now find lower bounds for the first two factors. Let $f_{n}(y)=\left(\frac{1-y^{2 n-1}}{1-y^{2 n+1}}\right)^{2}, y=\kappa \rho$, $y \in(0,1)$. The zeros of $f_{n}^{\prime}(y)$ in $(0,1)$ coincide with the zeros of $p(y)$ $=2 y^{2 n+1}-(2 n+1) y^{2}+(2 n-1)$. By Descartes's rule of signs, $p$ has either two positive roots or none. Since $p(1)=p^{\prime}(1)=0, p$ is nonzero in $(0,1)$ and hence 
so is $f_{n}^{\prime}$. It follows that, for $y \in(0,1), f_{n}(y)>\min \left\{f(0), \lim _{y \uparrow 1} f_{n}(y)\right\}=$ $\left(\frac{2 n-1}{2 n+1}\right)^{2}$. Note that $\left(\frac{2 n-1}{2 n+1}\right)^{2}$ is an increasing function of $n$.

Let $g_{n}(y)=\frac{1+y^{2 n+1}}{1+y^{2 n-1}}, y=\kappa \rho, y \in(0,1)$. The zeros of $g_{n}^{\prime}(y)$ in $(0,1)$ coincide with those of $q_{n}(y)=2 y^{2 n+1}+(2 n+1) y^{2}-(2 n-1)$. By Descartes's rule of signs and the intermediate value theorem, $q_{n}(y)$ has exactly one zero in $(0,1)$. Hence, so does $g_{n}^{\prime}(y)$. It is easily verified that this zero gives a minimum for $g_{n}(y)$.

Since $\frac{\partial g_{n}}{\partial n}>0$ for $y \in(0,1), g_{n}$ is an increasing function of $n$. Hence so is $\min _{n}\left\{g_{n}(y): y \in(0,1)\right\}$. In order to find a lower bound for $g_{n}(y)$, we consider $g_{2}(y)$ when $\kappa=4$ and $g_{3}(y)$ when $\kappa=2.19$.

The zero of $g_{2}^{\prime}(y)$ in $(0,1)$ is that of $q_{2}(y)=(y+1)^{2}\left(2 y^{3}-4 y^{2}+6 y-3\right)$. The cubic has the unique real root $y_{2}=\frac{1}{3}\left(2+\left(\frac{25}{2}\right)^{1 / 3}-(10)^{1 / 3}\right)$. Evaluating, one finds that $g_{2}\left(y_{2}\right)>0.8$. Hence, if $\kappa=4$ and $n \geqq 2$,

$$
\begin{aligned}
\frac{H_{n}(\kappa \rho)}{H_{n}(\rho)} & >\kappa \cdot \min _{y \in(0,1)} f_{2}(y) \cdot \min _{y \in(0,1)} g_{2}(y) \\
& >(4)\left(\frac{9}{25}\right)(0.8)>1 .
\end{aligned}
$$

To find the zero $y_{3}$ of $g_{3}^{\prime}$ in $(0,1)$, consider $q_{3}(y)=2 y^{7}+7 y^{2}-5$. Using Newton's method to approximate $y_{3}$, the intermediate value theorem to verify the approximation, and the mean value theorem to determine a lower bound for $g_{3}\left(y_{3}\right)$, one finds that $g_{3}\left(y_{3}\right)>0.9$. Thus for $\kappa=2.19$ and $n \geqq 3$,

$$
\begin{aligned}
\frac{H_{n}(\kappa \rho)}{H_{n}(\rho)} & >\kappa \cdot \min _{y \in(0,1)} f_{3}(y) \cdot \min _{y \in(0,1)} g_{3}(y) \\
& >(2.19)\left(\frac{25}{49}\right)(0.9)>1 . \square
\end{aligned}
$$

Lemma 2.4. If $\mu=0, H_{n}\left(\rho_{1}\right)>H_{n}\left(\rho_{4}\right)$ for $n \geqq 2$. If $\mu \neq 0, H_{n}\left(\rho_{2}\right)>H_{n}\left(\rho_{3}\right)$ for $n \geqq 2$ in regions II and III and for $n \geqq 3$ in region I.

Proof. If $\rho>\kappa \sigma$ with $\kappa>2$ and $\rho, \sigma \in(0,1)$, then $H_{n}(\rho)>H_{n}(\rho / \kappa)>H_{n}(\sigma)$ by Lemmas 2.3 and 2.1. The result follows by Lemma 2.2.

\section{Comparison of initial terms}

Let $F(x)=\frac{1-x^{2}}{2 x} G(\rho(x))$ and let $F_{n}(x)=\frac{1-x^{2}}{2 x} G_{n}(\rho(x))$. Note that $F_{n+1}(x)=H_{n}(\rho(x)) \cdot F_{n}(x)$; this will be used in Sect. 4 . In this section we prove several inequalities for $F_{1}, F_{2}$, and $F_{3}$.

Lemma 3.1. If $\mu=0,1+t^{2} F_{1}(\lambda)=s F_{1}(p / s)$. If $\mu \neq 0, F_{1}(\lambda)+t^{2} F_{1}(\mu)=s F_{1}(r / s)$. 
Proof. Recalling that $s=1+t^{2}$, the lemma follows immediately from

$$
F_{1}(x)=\frac{1-x^{2}}{2 x} \cdot 4 \cdot \frac{1-\sqrt{1-x^{2}}}{x} \cdot \frac{1+\frac{1}{x^{2}}\left(1-\sqrt{1-x^{2}}\right)^{2}}{\left[1-\frac{1}{x^{2}}\left(1-\sqrt{1-x^{2}}\right)^{2}\right]^{2}}=1
$$

We now express $F_{2}(x)$ as a rational function of $x$.

$$
\begin{aligned}
F_{2}(x) & =\frac{1-x^{2}}{2 x} \cdot 4 \cdot \frac{\left(1-\sqrt{1-x^{2}}\right)^{3}}{x^{3}} \cdot \frac{1+\frac{1}{x^{6}}\left(1-\sqrt{1-x^{2}}\right)^{6}}{\left[1-\frac{1}{x^{6}}\left(1-\sqrt{1-x^{2}}\right)^{6}\right]^{2}} \\
& =2 x^{2}\left(1-x^{2}\right)\left(1-\sqrt{1-x^{2}}\right)^{3} \cdot \frac{x^{6}+\left(1-\sqrt{1-x^{2}}\right)^{6}}{\left[x^{6}-\left(1-\sqrt{1-x^{2}}\right)^{6}\right.} \\
& =\frac{x^{2}\left(4-3 x^{2}\right)}{\left(4-x^{2}\right)^{2}}
\end{aligned}
$$

Lemma 3.2. If $\mu=0, t^{2} F_{2}(\lambda)>s F_{2}(p / s)$.

Proof. In this case we have $t=\frac{1}{2}$,

$$
t^{2} F_{2}(\lambda)=\frac{1}{4} \frac{\lambda^{2}\left(4-3 \lambda^{2}\right)}{\left(4-\lambda^{2}\right)^{2}}, \quad \text { and } \quad s F_{2}(p / s)=\frac{5}{4} \lambda^{2} \cdot \frac{100-3 \lambda^{2}}{\left(100-\lambda^{2}\right)^{2}} .
$$

Let $x=\lambda^{2} \neq 0$. Then showing that $t^{2} F_{2}(\lambda)>s F_{2}(p / s)$ is equivalent to showing that $f(x)>0$, where

$$
\begin{aligned}
f(x) & =(4-3 x)(100-x)^{2}-5(100-3 x)(4-x)^{2} \\
& =32,000-26,560 x-16 x^{2}+12 x^{3} .
\end{aligned}
$$

$f$ is obviously positive for $x \in(0,1)$.

The remaining lemmas in this section deal with the case $\mu \neq 0$. The idea of their proofs is identical to that of Lemma 3.2, but complicated by the appearance of $c$ and by the high degree and large coefficients of the polynomials which occur. The skeleton of all the proofs is the same: one shows that a polynomial $f(x, c)$ (where $x=\lambda^{2}$ ) is positive for $0<x<\frac{1}{c^{2}}$ and the appropriate domain for $c$. Essentially, either $f\left(\frac{1}{c^{2}}, c\right)>0$ and $\frac{\partial f}{\partial x}<0$, or one bounds $f$ by a function with such behavior. We indicate the polynomial which arises in each case and defer the elementary but tedious computations to the appendix.

Lemma 3.3. In region II, $t^{2} F_{2}(\mu)>s F_{2}(r / s)$.

Proof. Let $\mu=c \lambda, 1.6 \leqq c<10.0<\mu<1$ forces $0<\lambda<\frac{1}{c}$. Since $t=1$, $t^{2} F_{2}(\mu)=\frac{c^{2} \lambda^{2}\left(4-3 c^{2} \lambda^{2}\right)}{\left(4-c^{2} \lambda^{2}\right)^{2}}$. Recalling that $r=\mu t^{2}-\lambda$ and $s=1+t^{2}$, 
$s F_{2}(r / s)=\frac{2 \lambda^{2}(c-1)^{2}\left[16-3 \lambda^{2}(c-1)^{2}\right]}{\left[16-\lambda^{2}(c-1)^{2}\right]^{2}}$. Let $x=\lambda^{2}$. Then showing that $t^{2} F_{2}(\mu)$ $>s F_{2}(r / s)$ is equivalent to showing that the function

$$
f(x, c)=c^{2}\left(4-3 c^{2} x\right)\left[16-(c-1)^{2} x\right]^{2}-2(c-1)^{2}\left[16-3(c-1)^{2} x\right]\left(4-c^{2} x\right)^{2}
$$

is positive for $1.6 \leqq c<10$ and $0<x<\frac{1}{c^{2}}$.

Lemma 3.4. In region III, $t^{2} F_{2}(\mu)>s F_{2}(r / s)$.

Proof. Let $\mu=c \lambda, c \geqq 10$, and $t=\frac{1}{2}$. Then

$$
t^{2} F_{2}(\mu)=\frac{1}{4} \frac{c^{2} \lambda^{2}\left(4-3 c^{2} \lambda\right)}{\left(4-c^{2} \lambda^{2}\right)^{2}} \text { and } s F_{2}\left(\frac{r}{s}\right)=\frac{5}{4} \cdot \frac{\lambda^{2}(c-4)^{2}\left[100-3 \lambda^{2}(c-4)^{2}\right]}{\left[100-(c-4)^{2} \lambda^{2}\right]^{2}} .
$$

Let $x=\lambda^{2} \neq 0$. One shows that

$$
f(x, c)=c^{2}\left(4-3 c^{2} x\right)\left[100-(c-4)^{2} x\right]^{2}-5(c-4)^{2}\left[100-3 x(c-4)^{2}\right]\left(4-c^{2} x\right)^{2}
$$

is positive for $c \geqq 10$ and $0<x<\frac{1}{c^{2}}$.

Unfortunately, the analogue of Lemmas 3.3 and 3.4 fails' in region I. We prove instead the following two lemmas.

Lemma 3.5. In region $\mathrm{I}, F_{2}(\lambda)+t^{2} F_{2}(\mu)>s F_{2}(r / s)$.

Proof. Let $\mu=c \lambda, 1 \leqq c<1.6$, and $t=2$. Then

$$
F_{2}(\lambda)+t^{2} F_{2}(\mu)=\lambda^{2} \cdot \frac{\left(4-3 \lambda^{2}\right)\left(4-c^{2} \lambda^{2}\right)^{2}+4 c^{2}\left(4-3 c^{2} \lambda^{2}\right)\left(4-\lambda^{2}\right)^{2}}{\left(4-\lambda^{2}\right)^{2}\left(4-c^{2} \lambda^{2}\right)^{2}}
$$

and

$$
s F_{2}\left(\frac{r}{s}\right)=5(4 c-1)^{2} \lambda^{2} \frac{\left[100-3(4 c-1)^{2} \lambda^{2}\right]}{\left[100-(4 c-1)^{2} \lambda^{2}\right]^{2}}
$$

Let $w=\lambda^{2} \neq 0$. One shows that

$$
\begin{aligned}
f(x, c)= & {\left[(4-3 x)\left(4-c^{2} x\right)^{2}+4 c^{2}\left(4-3 c^{2} x\right)(4-x)^{2}\right]\left[100-(4 c-1)^{2} x\right]^{2} } \\
& -5(4 c-1)^{2}\left[100-3(4 c-1)^{2} x\right](4-x)^{2}\left(4-c^{2} x\right)^{2}
\end{aligned}
$$

is positive for $1 \leqq c<1.6$ and $0<x<\frac{1}{c^{2}}$.

We now express $F_{3}(x)$ as a rational function of $x$. A calculation using the identity $a^{5} \pm b^{5}=(a \pm b)\left(a^{4} \mp a^{3} b+a^{2} b \mp a b^{3}+b^{4}\right)$ shows that

$$
\begin{aligned}
& x^{10}+\left(1-\sqrt{1-x^{2}}\right)^{10}=2\left(1-\sqrt{1-x^{2}}\right)^{5}\left(5 x^{4}-20 x^{2}+16\right) \\
& x^{10}-\left(1-\sqrt{1-x^{2}}\right)^{10}=2 \sqrt{1-x^{2}}\left(1-\sqrt{1-x^{2}}\right)^{5}\left(x^{4}-12 x^{2}+16\right)
\end{aligned}
$$


and hence that

$$
\begin{aligned}
F_{3}(x) & =\frac{1-x^{2}}{2 x} \cdot 4 \cdot \frac{\left(1-\sqrt{1-x^{2}}\right)^{5}}{x^{5}} \frac{1+\frac{1}{x^{10}}\left(1-\sqrt{1-x^{2}}\right)^{10}}{\left[1-\frac{1}{x^{10}}\left(1-\sqrt{1-x^{2}}\right)^{10}\right]^{2}} \\
& =2 x^{4}\left(1-x^{2}\right)\left(1-\sqrt{1-x^{2}}\right)^{5} \frac{x^{10}+\left(1-\sqrt{1-x^{2}}\right)^{10}}{\left[x^{10}-\left(1-\sqrt{1-x^{2}}\right)^{10}\right]^{2}} \\
& =\frac{x^{4}\left(5 x^{4}-20 x^{2}+16\right)}{\left(x^{4}-12 x^{2}+16\right)^{2}} .
\end{aligned}
$$

Lemma 3.6. In region $\mathrm{I}, t^{2} F_{3}(\mu)>s F_{3}(r / s)$.

Proof. Let $\mu=c \lambda, 1 \leqq c<1.6$, and $t=2$. Then

and

$$
t^{2} F_{3}(\mu)=\frac{4 c^{4} \lambda^{4}\left(5 c^{4} \lambda^{4}-20 c^{2} \lambda^{2}+16\right)}{\left(c^{4} \lambda^{4}-12 c^{2} \lambda^{2}+16\right)^{2}}
$$

$$
s F_{3}\left(\begin{array}{l}
r \\
s
\end{array}\right)=5 \cdot \frac{\lambda^{4}(4 c-1)^{4}\left[5 \lambda^{4}(4 c-1)^{4}-500 \lambda^{2}(4 c-1)^{2}+10,000\right]}{\left[\lambda^{4}(4 c-1)^{4}-300 \lambda^{2}(4 c-1)^{2}+10,000\right]^{2}} .
$$

Let $x=\lambda^{2} \neq 0$. One shows that

$$
\begin{aligned}
f(x, c)= & 4 c^{4}\left(5 c^{4} x^{2}-20 c^{2} x+16\right)\left[(4 c-1)^{4} x^{2}-300(4 c-1)^{2} x+10^{4}\right]^{2} \\
- & 25(4 c-1)^{4}\left[(4 c-1)^{4} x^{2}-100(4 c-1)^{2} x+2,000\right] \\
& \left(c^{4} x^{2}-12 c^{2} x+16\right)^{2},
\end{aligned}
$$

is positive for $1 \leqq c<1.6$ and $0<x<\frac{1}{c^{2}}$.

\section{Proof of the theorem}

Theorem. Let $\mathscr{E}$ be an ellipsoid in $\mathbb{C}^{n}, n \geqq 2$, symmetric about 0 . Then $\mathscr{E}$ is biholomorphic to the ball in $\mathbb{C}^{n}$ if and only if its infinitesimal Kobayashi metric at 0 is hermitian.

Proof. It is well known that, if a domain is biholomorphic to the ball, its Kobayashi metric at any point is hermitian. For the other implication we assume that $\mathscr{E}$ is not biholomorphic to the ball. As discussed in Sect. 1, we may for simplicity and without loss of generality carry out the proof in $\mathbb{C}^{2}$. Recall that the ellipsoid is defined by

$$
|z|^{2}+|w|^{2}+\lambda \operatorname{Re}\left(z^{2}\right)+\mu \operatorname{Re}\left(w^{2}\right)<1
$$

with $\lambda \neq 0$. 
If $\mu=0$, consider the vectors $i t \frac{\partial}{\partial z}$ and $\frac{\partial}{\partial w}$ with $t=\frac{1}{2}$. Comparing (1.4), (1.5), and (1.6) with the definition of $F(x)$ in Sect. 3,

$$
\left\|i t \frac{\partial}{\partial z}\right\|^{2}+\left\|\frac{\partial}{\partial w}\right\|^{2}=1+t^{2} F(\lambda)=1+\sum_{n=1}^{\infty} t^{2} F_{n}(\lambda)
$$

and

$$
\left\|i t \frac{\partial}{\partial z}+\frac{\partial}{\partial w}\right\|_{\sim}^{2}=s F\left(\frac{p}{s}\right)=\sum_{n=1}^{\infty} s F_{n}\left(\frac{p}{s}\right) \text {. }
$$

We prove inductively that the series $\sum_{n=2}^{\infty} t^{2} F_{n}(\lambda)$ dominates the series $\sum_{n=2}^{\infty} s F_{n}(p / s)$ term-by-term. By Lemma 3.2, $t^{2} F_{2}(\lambda)>s F_{2}(p / s)$. Suppose that $t^{2} F_{k}(\lambda)>s F_{k}(p / s)$. Then

$$
t^{2} F_{k+1}(\lambda)=H_{k}(\rho(\lambda)) t^{2} F_{k}(\lambda)>H_{k}\left(\rho\left(\frac{p}{s}\right)\right) s F_{k}\left(\frac{p}{s}\right)=s F_{k+1}\left(\frac{p}{s}\right)
$$

by Lemma 2.4, completing the induction argument. Since $1+t^{2} F_{1}(\lambda)=s F_{1}(p / s)$ (Lemma 3.1) and the series are positive it follows that $1+\sum_{n=1}^{\infty} t^{2} F_{n}(\lambda)$ $>\sum_{n=1}^{\infty} s F_{n}(p / s)$. Hence

$$
\left\|i t \frac{\partial}{\partial z}\right\|^{2}+\left\|\frac{\partial}{\partial w}\right\|^{2}>\left\|i t \frac{\partial}{\partial z}+\frac{\partial}{\partial w}\right\|_{\sim}^{2} \geqq\left\|i t \frac{\partial}{\partial z}+\frac{\partial}{\partial w}\right\|^{2}
$$

and the parallelogram law fails.

The proof for the case $\mu \neq 0$ is essentially the same as for $\mu=0$. The vectors considered are $\frac{\partial}{\partial z}$ and $i t \frac{\partial}{\partial w}$ where $t=2,1$, and $\frac{1}{2}$ in regions I, II, and III respectively. From (1.1), (1.2), and (1.3)

and

$$
\left\|\frac{\partial}{\partial z}\right\|^{2}+\left\|i t \frac{\partial}{\partial w}\right\|^{2}=\sum_{n=1}^{\infty} F_{n}(\lambda)+\sum_{n=1}^{\infty} t^{2} F_{n}(\mu)
$$

$$
\left\|\frac{\partial}{\partial z}+i t \frac{\partial}{\partial w}\right\|_{-}^{2}=\sum_{n=1}^{\infty} s F_{n}\left(\frac{r}{s}\right) \text {. }
$$

It suffices to compare only $t^{2} F_{n}(\mu)$ and $s F_{n}(r / s)$ since $F_{n}(\lambda)$ is positive. In regions II and III, the induction begins at $n=2$ and uses Lemmas 3.3 and 3.4. In region III the induction begins at $n=3$, using Lemma 3.6; one then uses Lemma 3.5 to complete the argument. Failure of the parallelogram law results from

$$
\left\|\frac{\partial}{\partial z}\right\|^{2}+\left\|i t \frac{\partial}{\partial w}\right\|^{2}>\left\|\frac{\partial}{\partial z}+i t \frac{\partial}{\partial w}\right\|_{\sim}^{2} \geqq\left\|\frac{\partial}{\partial z}+i t \frac{\partial}{\partial w}\right\|^{2} .
$$

We now indicate briefly why the Kobayashi metric at 0 is hermitian if and only if the indicatrix is biholomorphic to the ball. If the metric is hermitian it 
gives an inner product on the tangent space. The linear map sending each element of an orthonormal basis to a standard basis vector in $\mathbb{C}^{n}$ is a biholomorphism between $I_{0}$ and the ball. For the other direction, suppose the indicatrix is biholomorphic to the ball. Then (since $I_{0}$ is circular), by $\mathrm{H}$. Cartan's uniqueness theorem the two are linearly equivalent. Define an inner product on the tangent space by pulling back the Euclidean inner product via the linear map. The norm induced by this inner product agrees with the Kobayashi norm.

\section{Appendix}

End of proof of Lemma 2.2. In region $\mathrm{I}, \quad 1 \leqq c<1.6, \quad t=2$, $\rho_{2}=\left(1-\sqrt{1-x^{2} \lambda^{2}}\right)^{2} /\left(c^{2} \lambda^{2}\right), \quad$ and $\quad \rho_{3}=\left(5-\sqrt{25-(4 c-1)^{2} \lambda^{2}}\right)^{2} /\left((4 c-1)^{2} \lambda^{2}\right)$. $\lim _{\lambda 10} \rho_{2} / \rho_{3}=25 c^{2} /(4 c-1)^{2}$. It is easily seen that this is minimized for $c=1.6$ and $\lambda \downarrow 0$

that the minimum is greater than 2.1904. For $\lambda \in(0,1 / c), \rho_{2}=2.1904 \rho_{3}$ if and only if

$$
\frac{1}{c^{2} \lambda^{2}}\left(1-\sqrt{1-c^{2} \lambda^{2}}\right)^{2}=\frac{1.48^{2}}{(4 c-1)^{2} \lambda^{2}}\left(5-\sqrt{25-(4 c-1)^{2} \lambda^{2}}\right)
$$

or, equivalently, $P_{I}(\lambda, c)=0$ where

$$
\begin{aligned}
P_{I}(\lambda, c) & =\lambda^{2}\left(1.417052 c^{2}(4 c-1)^{2} \lambda^{2}+8.7616+24.34304 c-18.51776 c^{2}\right) \\
& \geqq \lambda^{2}\left(1.417052 c^{2}(4 c-1)^{2} \lambda^{2}+8.7616-5.285376 c\right) \\
& \geqq \lambda^{2}\left(1.417052 c^{2}(4 c-1)^{2} \lambda^{2}+0.3049984\right) .
\end{aligned}
$$

The inequalities follow from the fact that $c \leqq 1.6$. Thus $P_{I}(\lambda, c)>0$. Hence $\rho_{2}>2.19 \rho_{3}$ for $\lambda \in(0,1 / c), c \in[1,1.6)$.

Proof of Lemma 3.3. It suffices to show that, for fixed $c \in[1.6,10], f\left(\frac{1}{c^{2}}, c\right)>0$ and $\frac{\partial f}{\partial x}<0$ for $x \in\left(0, \frac{1}{c^{2}}\right) . f\left(\frac{1}{c^{2}}, c\right)=\frac{1}{c^{2}} F(c)$, where $F(c)=-9 c^{4}+420 c^{3}$ $+10 c^{2}-220 c+55$. But

$$
\begin{aligned}
F(c) & =c^{3}(-9 c+420)+10 c^{2}-220 c+55 \\
& \geqq 330 c^{3}+10 c^{2}-220 c+55=10 c^{2}+c\left(330 c^{2}-220\right)+55 \\
& \geqq 10 c^{2}+624 c+55>0,
\end{aligned}
$$

where the first inequality follows from $c \leqq 10$ and the second from $c \geqq 1.6$. Hence $f\left(\frac{1}{c^{2}}, c\right)>0$. Expanding $f(x, c)$ and differentiating,

$$
\begin{aligned}
\frac{\partial f}{\partial x}= & -544 c^{4}-640 c^{3}+704 c^{2}-384 c+96 \\
& +2 c^{2}\left(20 c^{4}+48 c^{3}-200 c^{2}+176 c-44\right) x \\
& +3 c^{4}\left(3 c^{4}-12 c^{3}+18 c^{2}-12 c+3\right) x^{2}
\end{aligned}
$$


The coefficient of $x^{2}$ is $A(c)=9 c^{4}(c-1)^{4}>0$ for $c \in[1.6,10]$. Hence, for

$$
\begin{aligned}
& x \in\left(0, \frac{1}{c^{2}}\right), \frac{\partial f}{\partial x}<\max \left\{\left.\frac{\partial f}{\partial x}\right|_{x=0},\left.\frac{\partial f}{\partial x}\right|_{x=\frac{1}{c^{2}}}\right\} . \\
&\left.\frac{\partial f}{\partial x}\right|_{x=0}=-544 c^{4}-640 c^{3}+704 c^{2}-384 c+96 \\
&=32\left(-17 c^{4}-20 c^{3}+22 c^{2}-12 c+3\right) \\
&<32\left(-17 c^{4}+2 c^{2}-9\right) \\
&<32\left(-15 c^{2}-9\right)<0 \\
&\left.\frac{\partial f}{\partial x}\right|_{x=\frac{1}{c^{2}}}=-495 c^{4}-58 c^{3}+358 c^{2}-68 c+17 \\
&<-495 c^{4}-222 c^{2}-51<0
\end{aligned}
$$

where the inequalities follow from $c>1$. Hence $\frac{\partial f}{\partial x}>0$. Therefore $f(x, c)>0$ for $c \in[1.6,10)$ and $x \in\left(0, \frac{1}{c^{2}}\right)$.

Proof of Lemma 3.4.

$$
\begin{aligned}
f\left(\frac{1}{c^{2}}, c\right) & =\frac{1}{c^{2}}\left(5,436 c^{4}+35,424 c^{3}-62,144 c^{2}-34,816 c+34,816\right) \\
& \geqq \frac{1}{c^{2}}\left(5,436 c^{4}+2,886,144 c+39,816\right)>0,
\end{aligned}
$$

where the inequality follows from $c \geqq 10$. Expanding $f(x, c)$ and differentiating,

$$
\begin{aligned}
\frac{\partial f}{\partial x}= & \left(61,440-61,440 c+74,240 c^{2}-29,440 c^{3}-26,560 c^{4}\right) \\
& +2 c^{2}\left(-29,696+29,696 c-9,536 c^{2}+1,056 c^{3}-16 c^{4}\right) x \\
& +3 c^{4}\left(3,072-3,072 c+1,152 c^{2}-192 c^{3}+12 c^{4}\right) x^{2} .
\end{aligned}
$$

The coefficient of $x^{2}$ is $A(c)=36 c^{4}(c-4)\left(c^{3}-12 c^{2}+48 c-64\right)$. It is easily verified that the cubic factor has only one real root, which lies in $(0,10)$. Hence $A(c)$ does not change sign for $c \geqq 10$, and $\lim _{c \rightarrow \infty} A(c)>0$ implies $A(c)>0$ for $c \geqq 10$.

Hence, for $x \in\left(0, \frac{1}{c^{2}}\right), \frac{\partial f}{\partial x}<\max \left\{\left.\frac{\partial f}{\partial x}\right|_{x=0},\left.\frac{\partial f}{\partial x}\right|_{x=\frac{1}{c^{2}}}\right\}$.

$$
\begin{aligned}
\left.\frac{\partial f}{\partial x}\right|_{x=0} & =61,440-614,400 c+74,240 c^{2}-29,440 c^{3}-26,560 c^{4} \\
& \leqq-552,960-220,160 c^{2}-26,560 c^{4}<0 \\
\left.\frac{\partial f}{\partial x}\right|_{x=\frac{1}{c^{2}}} & =11,264-11,264 c+58,624 c^{2}-27,904 c^{3}-26,556 c^{4} \\
& \leqq-101,376-220,416 c^{2}-26,556 c^{4}<0
\end{aligned}
$$


The inequalities follow from the fact that $c \geqq 10$. Hence $\frac{\partial f}{\partial x}<0$ and $f(x, c)>0$ for $c \geqq 10$ and $x \in\left(0, \frac{1}{c^{2}}\right)$.

Proof of Lemma 3.5. The method is similar to that of Lemmas 3.3 and 3.4, except that the derivative to be shown negative is cubic rather than quartic in $x$. Instead of dealing with it directly we majorize it by a polynomial in $c$.

$$
\begin{aligned}
f\left(\frac{1}{c^{2}}, c\right) & =\frac{16}{c^{6}}\left(\begin{array}{l}
-9,216 c^{8}+6,816 c^{7}+28,528 c^{6}-2,608 c^{5} \\
-16,600 c^{4}-766 c^{3}+611 c^{2}-112 c+7
\end{array}\right) \\
& \geqq \frac{16}{c^{3}}\left(-9,216 c^{5}+6,816 c^{4}+28,528 c^{3}-2,608 c^{2}-16,600 c-766\right) \\
& \geqq \frac{16}{c^{2}}\left(-9,216 c^{4}+6,816 c^{3}+28,528 c^{2}-2,608 c-17,336\right) \\
& =\frac{128}{c^{2}}\left(-1,152 c^{4}+852 c^{3}+3,566 c^{2}-326 c-2,167\right)
\end{aligned}
$$

$g(c)=-1,152 c^{4}+852 c^{3}+3,566 c^{2}-326 c-2,167$ has only two real roots, of which one is less than 1 and one is greater than 1.6. Hence $g(c)$ does not change sign on $[1,1.6)$; since $g(1)>0$, it follows that $g(c)>0$ on $[1,1.6]$. Hence the same is true of $f\left(\frac{1}{c^{2}}, c\right)$.

$$
\begin{aligned}
\frac{\partial f}{\partial x}= & \left(-424,960-471,040 c-399,360 c^{2}-1,085,440 c^{3}-732,160 c^{4}\right) \\
& +\left(\begin{array}{c}
-512+33,792 c+491,520 c^{2}+1,003,520 c^{3}+791,040 c^{4} \\
+365,568 c^{5}+120,832 c^{6}
\end{array}\right) x \\
& +\left(\begin{array}{c}
576-9,216 c+45,696 c^{2}-89,856 c^{3}-312,000 c^{4}-232,704 c^{5} \\
-139,776 c^{6}-36,864 c^{7}+36,864 c^{8}
\end{array}\right) x^{2} \\
& +\left(-320 c^{2}+5,120 c^{3}-20,800 c^{4}+3,200 c^{5}+70,400 c^{6}\right. \\
& \left.+20,480 c^{7}-20,480 c^{8}\right) x^{3} \\
= & A_{0}(c)+A_{1}(c) x+A_{2}(c) x^{2}+A_{3}(c) x^{3} .
\end{aligned}
$$

We now find a function $h(c)$ such that $\frac{\partial f}{\partial x}<h(c)<0$ for $c \in[1,1.6), x \in\left(0, \frac{1}{c^{2}}\right)$. Consider first

$$
\begin{aligned}
A_{3}(c)-70,400 c^{6} & =320 c^{2}\left(-1+16 c-65 c^{2}+10 c^{3}+64 c^{5}-64 c^{6}\right) \\
& \leqq 320 c^{2}\left(-1+16 c-65 c^{2}+10 c^{3}\right) \\
& \leqq 320 c^{2}\left(-1+16 c-49 c^{2}\right) \\
& \leqq 320 c^{2}(-1-33 c)<0
\end{aligned}
$$


where $c \leqq 1.6$ was used in the second inequality and $c \geqq 1$ in the first and third.

Since $x<\frac{1}{c^{2}} \leqq 1, \quad 70, \quad 400 c^{6} x^{3}<70,400$. Hence $\frac{\partial f}{\partial x}<A_{0}(c)+70,400$ $+A_{1}(c) x+A_{2}(c) x^{2}$. We now consider $A_{2}(c)$.

$$
\begin{aligned}
A_{2}(c)= & 64\left(9-144 c+714 c^{2}-1,404 c^{3}-4,875 c^{4}-3,636 c^{5}-2,184 c^{6}\right. \\
& \left.-576 c^{7}+576 c^{8}\right) \\
< & 64\left(9-144 c+714 c^{2}-1,404 c^{3}-4,875 c^{4}-3,636 c^{5}\right. \\
& \left.-2,184 c^{6}+345 c^{7}\right) \\
\leqq & 64\left(-135-690 c^{2}-4,875 c^{4}-3,636 c^{5}-1,632 c^{6}\right)<0,
\end{aligned}
$$

where $c \leqq 1.6$ was used in the first inequality, and $1 \leqq c<1.6$ in the second. Hence $\frac{\partial f}{\partial x}<A_{0}(c)+70,400+A_{1}(c) x$, and we consider $A_{1}(c) x$.

$$
\begin{aligned}
A_{1}(c) x< & \left(33,792 c+491,520 c^{2}+1,003,520 c^{3}+791,040 c^{4}\right. \\
& \left.+365,568 c^{6}+120,832 c^{6}\right) x \\
< & 525,312+1,003,520 c+791,040 c^{2}+365,568 c^{3}+120,832 c^{4},
\end{aligned}
$$

where in the first inequality we used the positivity of $x$, and in the second that $x<\frac{1}{c^{2}} \leqq 1$. Hence

$$
\begin{aligned}
\frac{\partial f}{\partial x} & <A_{0}(c)+70,400+525,312+1,003,520 c+791,040 c^{2}+365,568 c^{3}+120,832 c^{4} \\
& =170,752+532,480 c+391,680 c^{2}-719,872 c^{3}-611,328 c^{4} \\
& =h(c) .
\end{aligned}
$$

Using the fact that $c \geqq 1$, it is easily seen that $h(c) \leqq-236,288$. Hence $\frac{\partial f}{\partial x}<0$ for $c \in[1,1.6), x \in\left(0, \frac{1}{c^{2}}\right)$.

Proof of Lemma 3.6. The method is similar to that of the preceding lemmas, except that $f(x, c)$ has much larger coefficients and higher total degree. In order to show that it is positive we estimate it from below by a more tractable polynomial. We may write $f(x)=a(x, c)-b(x, c)$, where

$$
\begin{aligned}
& a(x, c)=4 c^{4}\left(5 c^{4} x^{2}-20 c^{2} x+16\right)\left[(4 c-1)^{4} x^{2}-300(4 c-1)^{2} x+10^{4}\right]^{2} \\
& b(x, c)=25(4 c-1)^{4}\left[(4 c-1)^{4} x^{2}-100(4 c-1)^{2} x+2,000\right]\left(c^{4} x^{2}-12 c^{2} x+16\right)^{2} .
\end{aligned}
$$

We will now find $\tilde{a}(x, c)$ and $\tilde{b}(x, c)$ such that $a(x, c)>\tilde{a}(x, c), b(x, c)<\tilde{b}(x, c)$, and $\tilde{a}(x, c)-\tilde{b}(x, c)>0$.

Clearly $\quad a(x, c)>4 c^{4}\left(5 c^{4} x^{2}-20 c^{2} x+16\right)\left(10,000-300(4 c-1)^{2} x\right)^{2}$. Let $\alpha(x, c)=10,000-300(4 c-1)^{2} x . \alpha>0$ for $c \in[1,1.6], x \in\left[0, \frac{1}{c^{2}}\right] ; \alpha$, and hence 
$\alpha^{2}$, is minimized when $x=\frac{1}{c^{2}}$ and $c=1.6$. We use the cruder lower bound $\alpha^{2}>43,320,000$ and let $\tilde{a}(x, c)=17,328 \cdot 10^{4} c^{4}\left(5 c^{4} x^{2}-20 c^{2} x+16\right)$.

Let $\beta(x, c)=(4 c-1)^{4} x^{2}-100(4 c-1)^{2} x+2,000$. It is easily seen that $\beta<2,000$ for $c \in[1,1.6]$ and $x \in\left[0, \frac{1}{c^{2}}\right]$. Let $\tilde{b}(x, c)=5 \cdot 10^{4}(4 c-1)^{4}\left(c^{4} x^{2}-12 c^{2} x+16\right)^{2}$. Then $f(x, c)>\tilde{a}(x, c)-\tilde{b}(x, c)=10^{4} \tilde{f}(x, c)$ where

$$
\begin{gathered}
\tilde{f}(x, c)=17,328 c^{4}\left(5 c^{4} x^{2}-20 c^{2} x+16\right)-5(4 c-1)^{4}\left(c^{4} x^{2}-12 c^{2} x+16\right)^{2} . \\
\tilde{f}\left(\frac{1}{c^{2}}, c\right)=-125+2,000 c-12,000 c^{2}+32,000 c^{3}-14,672 c^{4} \\
>200 c\left(9-60 c+160 c^{2}-74 c^{3}\right) .
\end{gathered}
$$

The cubic is positive at $c=1$ and $c=1.6$ and has negative second derivative for $c \geqq 1$. Hence $\tilde{f}\left(\frac{1}{c^{2}}, c\right)>0, c \in[1,1.6]$.

We now show that $\frac{\partial f}{\partial x}<0$ for $c \in[1,1.6], x \in\left(0, \frac{1}{c^{2}}\right)$.

$$
\begin{aligned}
\frac{\partial \tilde{f}}{\partial x}= & 1,920 c^{2}-30,720 c^{3}+184,320 c^{4}-491,520 c^{5}+144,960 c^{6} \\
& +\left(-1,760 c^{4}+28,160 c^{5}-168,960 c^{6}+450,560 c^{7}-277,280 c^{8}\right) x \\
& +\left(360 c^{6}-5,760 c^{7}+34,560 c^{8}-92,160 c^{9}+92,160 c^{10}\right) x^{2} \\
& +\left(-20 c^{8}+320 c^{9}-1,920 c^{10}+5,120 c^{11}-5,120 c^{12}\right) x^{3} .
\end{aligned}
$$

Denote by $A(c)$ the coefficient of $x^{3}$. It follows from $c \geqq 1$ that $A(c)$ $\leqq c^{8}(-20-1,600 c), \quad$ so $\quad \frac{\partial \tilde{f}}{\partial x}<\frac{\partial \tilde{f}}{\partial x}-A(c) x^{3}$. Let $g(x, c)=\frac{1}{c^{2}}\left(\frac{\partial f}{\partial x}-A(c) x^{3}\right)$. Then

$$
\begin{aligned}
g(0, c) & =1,920-30,720+184,320 c^{2}-491,520 c^{3}+144,960 c^{4} \\
& \leqq 1,920-20,720 c+184,320 c^{2}-259,584 c^{3} \\
& \leqq-28,800-75,264 c^{2}<0,
\end{aligned}
$$

where the first inequality follows from $c \leqq 1.6$ and the second from $c \geqq 1$.

$$
\begin{aligned}
g\left(\frac{1}{c^{2}}, c\right) & =520-8,320 c+49,920 c^{2}-133,120 c^{3}-40,164 c^{4} \\
& \leqq-7,800-83,200 c^{2}-40,160 c^{4}<0
\end{aligned}
$$

since $c \geqq 1$. Denote by $B(c)$ the coefficient of $x^{2}$ in $g(x, c)$.

$$
\begin{aligned}
B(c) & =c^{4}\left(360-5,760 c+34,460 c^{2}-92,160 c^{3}+92,160 c^{4}\right) \\
& \geqq c^{4}(360+28,800)>0
\end{aligned}
$$


since $c \geqq 1$. It follows that $g(x, c)<0$, hence $\frac{\partial \tilde{f}}{\partial x}<0$, for $x \in\left(0, \frac{1}{c^{2}}\right)$. Therefore $\tilde{f}(x, c)>0$ and $f(x, c)>0$ for $c \in[1,1.6)$ and $x \in\left(0, \frac{1}{c^{2}}\right)$.

\section{References}

1. Jacobi, C.G.J.: Gesammelte Werke, vol. 1, New York: Chelsea 1969

2. Lempert, L.: La métrique de Kobayashi et la représentation des domaines sur la boule. Bull. Soc. math. Fr. 109, 427-474 (1981)

3. Lempert, L.: Holomorphic retracts and intrinsic metrics in convex domains. Anal. Math. 8, 257-261 (1982)

4. Lempert, L.: Holomorphic invariants, normal forms, and the moduli space of convex domains. Ann. Math. 128, 43-78 (1988)

5. Nehari, Z.: Conformal mapping. New York: Dover 1975

6. Obreschkoff, N.: Verteilung und Berechnung der Nullstellen reeller Polynome. Berlin: VEB Deutscher Verlag der Wissenschaften 1963

7. Reiffen, H.-J.: Die differentialgeometrischen Eigenschaften der invarianten Distanzfunktion von Caratheodory. Schriftenr. Math. Inst. Univ. Münster, 2. Ser. 26 (1963)

8. Royden, H.L.: Remarks on the Kobayashi metric. In: Several complex variables,II (Proc. Intern. Conf. Univ. of Maryland, 1970). (Lect. Notes Math., vol. 185, pp. 125-137) Berlin Heidelberg New York: Springer 1971

9. Rudin, W.: Function theory in the unit ball of $\mathbb{C}^{n}$. Berlin Heidelberg New York: Springer 1980

10. Sibony, N.: Remarks on the Kobayashi metric. Preprint

11. Tannery, J., Molk, J.: Eléments de la théorie des functions elliptiques, Tome IV. New York: Chelsea 1972

12. Webster, S.M.: On the mapping problem for algebraic real hypersurfaces. Invent. Math. 43, 53-68 (1977) 